\title{
Gasless laparoendoscopic single-site surgery with intraoperative autologous blood transfusion for management of ectopic pregnancy with significant hemoperitoneum: a retrospective observational study
}

\author{
Akihiro Takeda* (D), Shiori Tsuge, Mayu Shibata, Sanae Shinone and Hiromi Nakamura
}

\begin{abstract}
Background: With advances in diagnostic and therapeutic modalities, earlier detection of ectopic pregnancy with minimal symptoms makes laparoscopic management more common. However, if diagnostic delay occurs, significant hemoperitoneum associated with ectopic pregnancy is still a potentially life-threatening condition, which presents a therapeutic challenge especially when minimally invasive surgical approach is a significant concern. Herein, retrospective observational study in 18 consecutive cases of ectopic pregnancy with significant hemoperitoneum $\geq$ $800 \mathrm{~mL}$ was performed to assess the feasibility and efficacy of gasless laparoendoscopic single-site (LESS) surgery with intraoperative autologous blood cell salvage and donation for management.
\end{abstract}

Results: At triage, median shock index was 0.95, median hemoglobin value was $6.5 \mathrm{~g} / \mathrm{dL}$, and median serum $\beta-\mathrm{hCG}$ value was 13,651 U/L. Surgical diagnosis included 7 ampullary (including 5 abortions and 2 ruptures), 5 ruptured isthmic (including 1 heterotopic pregnancy in remnant tube after salpingectomy and 1 remnant tube pregnancy after salpingo-oophorectomy), 4 ruptured interstitial (including 1 case after salpingectomy), and 2 ruptured ovarian pregnancies. Among these cases, 16 cases were successfully managed by LESS surgery, while, in 2 cases with interstitial pregnancy, conversion to multi-port laparoscopic surgery along with transfusion of bank blood were required. Laparotomic conversion was not experienced. Surgical procedures in 16 cases successfully completed by LESS surgery with intraoperative autologous blood transfusion included unilateral salpingectomy for 7 ampullary and 5 isthmic pregnancies, cornuotomy and cornual resection for each 1 case with interstitial pregnancy, and unilateral salpingooophorectomy and partial ovarian resection for each 1 case with ovarian pregnancy. Median surgical duration was $57.5 \mathrm{~min}$, median amounts of salvaged peritoneal blood were $950 \mathrm{~mL}$ (range 800-2000 mL), and median amounts of transfused autologous blood were $540 \mathrm{~mL}$ (range 450-1300 mL). Major complications associated with LESS surgery and intraoperative autologous blood transfusion were not experienced.

Conclusion: Gasless LESS surgery with intraoperative autologous blood transfusion is a feasible minimally invasive surgical option with avoidance to use homologous blood for management of selected cases of ectopic pregnancy even with significant hemoperitoneum.

Keywords: Ectopic pregnancy, Hemoperitoneum, Intraoperative autologous blood transfusion, Laparoendoscopic single-site surgery

\footnotetext{
* Correspondence: gyendoscopy@gmail.com

Department of Obstetrics and Gynecology, Gifu Prefectural Tajimi Hospital,

5-161 Maebata-cho, Tajimi, Gifu 507-8522, Japan
} 


\section{Background}

With advances in diagnostic and therapeutic modalities, earlier detection of ectopic pregnancy with minimal symptoms becomes more common [1]. However, if diagnostic delay occurs, significant hemoperitoneum associated with ectopic pregnancy is still a potentially life-threatening condition $[1,2]$.

Traditionally, such morbidities have usually been managed by laparotomy, occasionally with homologous blood transfusion if necessary [3]. Along with progress in laparoscopic surgical techniques and expertise, improvement in perioperative management of hemodynamic condition by anesthesiologists has facilitated minimally invasive surgical management by laparoscopy for women experiencing hemodynamic instability due to ectopic pregnancy even with massive hemoperitoneum $[1,2,4,5]$.

Furthermore, intraoperative transfusion of autologous red blood cells retrieved from peritoneal cavity by specialized cell salvage device $[2,6,7]$ may negate the needs of homologous blood transfusion in selected cases if cardiopulmonary stability and coagulation status can be maintained.

To optimize the benefits of minimally invasive procedures in laparoscopic surgery, gynecologic surgeons have made attempts to reduce abdominal wall trauma by minimizing the size and/or decreasing the number of ports. Consequently, transumbilical laparoendoscopic single-site (LESS) surgery is rapidly evolving as a surgical procedure, in which single umbilical skin incision eventually conceals within the umbilicus, and its application is under active investigation in gynecological surgeries $[8,9]$.

Previous experiences indicated that ectopic pregnancy could be an acceptable candidate for management by LESS surgery with securing surgical view by gasless abdominal wall-lifting [8] or pneumoperitoneum [10], even under significant hemoperitoneum [8]. However, reports on the wider application of LESS surgery for such morbidities are limited at the present time [11]. Furthermore, feasibility of intraoperative autologous blood cell salvage and donation has never been shown in case series under setting with LESS surgery.

In the present report, the potential safety and efficacy of gasless LESS surgery with intraoperative autologous blood cell salvage and donation [2, 6, 7] were evaluated as key interventions for management of ectopic pregnancies with significant hemoperitoneum experienced at regional tertiary referral center.

\section{Methods}

\section{Patient selection}

Retrospective observational study was performed for 18 women experiencing ectopic pregnancy with significant hemoperitoneum $\geq 800 \mathrm{~mL}$. They were managed by gasless
LESS surgery with intraoperative autologous blood cell salvage and donation.

\section{Preoperative management}

When a patient with ectopic pregnancy was transferred to the emergency department of our hospital, anesthesiologist of the intensive care unit assessed and managed the patient's hemodynamic condition, while a gynecological examination was performed with measurement of serum $\beta$-human chorionic gonadotropin (hCG) value. If hemodynamic instability was assumed [2, 9], large-bore venous access was obtained, and rapid fluid resuscitation was initiated.

While rapid fluid administration was performed, the patient's hemodynamic status was precisely evaluated and the patients were classified into responders, transient responders, or non-responders. Laparoscopic surgery was conducted primarily for responders and transient responders. After an initial evaluation by ultrasonography, computerized tomography was performed to identify the extravasation site (Fig. 1a) before transferring to the operation room except for poor responders.

\section{Surgical procedures}

Gasless LESS was performed by the abdominal wall-lift method (Fig. 1b) in the patient with a low Trendelenburg position, under general endotracheal anesthesia [8, 12]. The surgical procedures were performed in a similar manner with standard laparoscopic instruments.

A $2.5-\mathrm{cm}$ midline umbilical skin incision was made vertically and carried down into the peritoneum. An Alexis wound retractor (X-small, Applied Medical, Rancho Santa Margarita, CA, USA) was placed in position through the umbilical incision with the bottom green ring inside the abdomen (Fig. 1b, arrowhead). After securing the peritoneal working space by an abdominal wall-lifting device (Mizuho Co., Tokyo, Japan) (Fig. 1b, arrow) [12], rigid $30^{\circ}$, 5-mm EndoEYE laparoscope (Olympus, Tokyo, Japan) was introduced and the entire abdominal cavity was inspected. Under isobaric continuous laparoscopic observation, multiple instruments could be introduced transumbilically through a wound retractor $[8,12]$.

When significant hemoperitoneum was confirmed under laparoscopic vision (Fig. 1c), the Continuous Autotransfusion System (Fresenius Kabi, Bad Homburg, Germany) was operated for intraoperative blood cell salvage $[2,8,9]$ (Fig. 1d). To obtain an optimal surgical view and retrieve the blood for autologous blood transfusion, pooled blood in the peritoneal cavity was irrigated, suctioned, and sent to the reservoir. Stored blood was centrifugally separated to make concentrated red blood cell solution with $40 \%$ hematocrit. Processed 

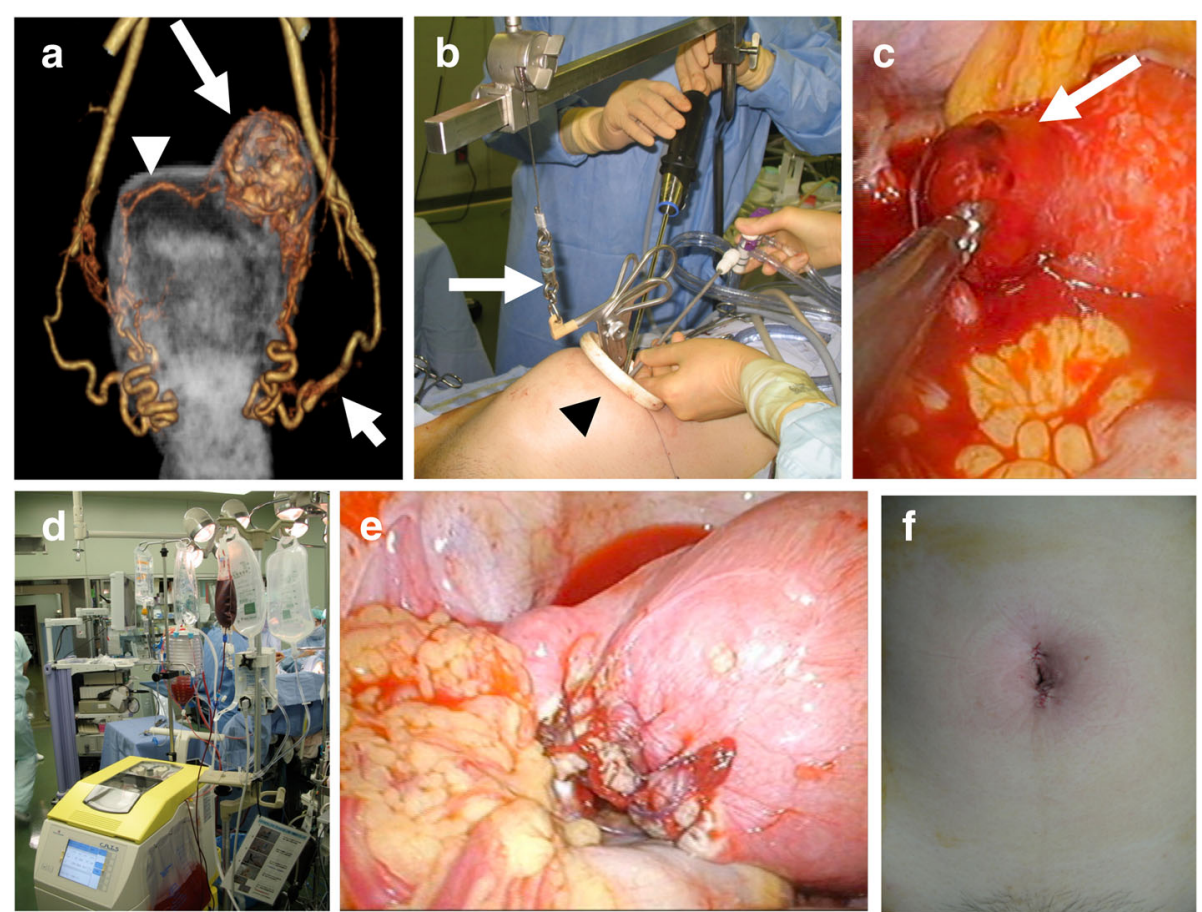

Fig. 1 Ruptured interstitial pregnancy managed by laparoendoscopic single-site cornuotomy with intraoperative autologous blood cell salvage and donation. A 29-year-old woman (gravida 3, para 2) was transferred under the suspicion of ectopic pregnancy at estimated 7 weeks of gestation. The serum $\beta$-human chorionic gonadotropin value was $50,736 \mathrm{mlU} / \mathrm{mL}$, and the shock index was 0.88 . The hemoglobin value was $6.5 \mathrm{~g} / \mathrm{dL}$. a A coronal view of three-dimensional computerized tomographic angiography showing a prominent vascular mass with extravasation (long arrow) supplied from the ascending branch of the left uterine artery (short arrow) with an anastomosing right uterine artery (arrowhead) in the left cornual region, indicating ruptured interstitial pregnancy. b Surgical working space secured by intra-abdominal fan retractor system (arrow) combined with wound retractor (arrowhead). c Emergency laparoendoscopic single-site surgery showing ruptured left interstitial pregnancy (arrow) with massive hemoperitoneum. d A Continuous Autotransfusion System (Fresenius Kabi, Bad Homburg, Germany) utilized for intraoperative blood cell salvage. e Hemostasis achieved by intracorporeal suturing and extracorporeal knot-tying after removal of the gestational products. $\mathbf{f}$ Single surgical wound limited to the umbilicus after skin closure. The amount of intraperitoneal bleeding was $1000 \mathrm{~mL}$, and $505 \mathrm{~mL}$ of retrieved blood was transfused back to the patient. The hemoglobin value on postoperative day 1 was $10.0 \mathrm{~g} / \mathrm{dL}$. Postoperatively, the systemic administration of methotrexate was required three times due to persistent ectopic pregnancy

blood was immediately transfused back to the patient through a microaggregate blood filter.

For salpingectomy, in case of ampullary and isthmic pregnancies, the mesosalpinx and proximal tubal stump were sealed and cut by LigaSure Atlas (Covidien Japan, Tokyo, Japan). For interstitial pregnancy, either cornuotomy (Fig. 1) or cornual resection was performed. In cornuotomy, gestational products (Fig. 1c, arrow) were removed by blunt and sharp dissection, followed by intracorporeal suturing by an Endo Stitch suturing device (Covidien Japan, Tokyo, Japan) and extracorporeal knot-tying (Fig. 1e), while cornual resection was performed by Harmonic Ace (Ethicon Japan, Tokyo, Japan), followed by suturing hemostasis.

In salpingo-oophorectomy for ovarian pregnancy, the infundibulopelvic and utero-ovarian ligaments were sealed and cut by LigaSure Atlas. In partial ovarian resection for ovarian pregnancy, gestational products were excised by blunt and sharp dissection, followed by suturing hemostasis. Local methotrexate (MTX) (50 mg/body) injection was added if residual tissue of ectopic pregnancy was concerned in cases with interstitial and isthmic pregnancies based on each surgeon's decision.

If contained extraction was appropriate, the excised tissue was put into a retriever bag and removed from the body through the umbilical port. After extensive washing with saline, the abdominal cavity was finally inspected for hemostasis and adjacent organ injury, followed by closure of umbilical wound (Fig. 1f). At the end of the procedure, amounts of peritoneal blood retrieved during surgery were estimated by measuring the volume of intraoperative suction, then subtracting the volume of liquid utilized for intraperitoneal washing.

After surgery, the patient was admitted to the intensive care unit for immediate postoperative management of the general condition. Occurrence of immediate postoperative complications was carefully monitored. A complete blood cell count was obtained on postoperative day 1 to determine the degree of anemia. 
When hemodynamic stability was achieved, the patient was moved to the gynecology ward. The patient received daily intravenous injections of iron supplement to treat anemia. C-reactive protein (CRP) measurement and a complete blood cell count were further performed on postoperative day 3 to confirm the absence of infection.

If postoperative infection was suspected by an elevated CRP value and/or elevated white blood cell count, extended administration of antibiotics was considered. After surgery, serum $\beta$-hCG value was serially measured every other day until the titers decreased to $50 \%$ of the pre-treatment levels and weekly thereafter, until the titers returned to normal values $(<6 \mathrm{mIU} / \mathrm{mL})$. If the disappearance of serum $\beta$-hCG was nonlinear when plotted on a semi-logarithmic graph, a significant delay of decline of $\beta$-hCG value was judged and MTX ( $50 \mathrm{mg} /$ body) was systemically administered under the diagnosis of persistent ectopic pregnancy [2, 8].

Patients were discharged, when they were fully ambulatory and apyrexial, in addition to confirmation of the decrease in serum $\beta$-hCG level and elevation in hemoglobin value above $7-8 \mathrm{~g} / \mathrm{dl}$. Patients were checked for early postoperative complications including postoperative infection, ileus, and persistent ectopic pregnancy occurring within 30 days after surgery.

\section{Clinical data analysis}

The clinical backgrounds, surgical diagnosis, procedures, and outcome measures were analyzed. The primary outcome was the successful gasless LESS surgical management with intraoperative autologous blood cell salvage and donation, without conversion to multi-port laparoscopic surgery and/or needs of homologous blood transfusion.

All statistical analyses were performed with Microsoft Excel 2011 for Mac OS X (Redmond, WA, USA) using the Statcel 3 add-in software program (OMS Pub. Inc., Saitama, Japan). First, variables were tested for normality using the Kolmogorov-Smirnoff test. Because variables in the present study were not normally distributed, results were expressed as the median value and range (minimum-maximum).

Shock index (SI) was calculated by dividing the triage heart rate by triage systolic blood pressure [2]. Patients were classified according to their initial SI (class I, SI $<0.6$; class II, SI $\geq 0.6$ to $<1.0$; class III, SI $\geq 1.0$ to $<1.4$; class IV, SI $\geq 1.4$ ). The Pearson coefficient of correlation was calculated between shock index and the amount of hemoperitoneum at surgical exploration. The probability value of correlation was calculated by Fisher $r$-to- $z$ transformation for Pearson correlation and a probability value of $<0.05$ was considered statistically significant.

\section{Results}

Among 167 ectopic pregnancies managed by gasless LESS surgery between 2010 and August 2018, intraoperative autologous blood cell salvage and donation were performed for 18 cases with significant hemoperitoneum $\geq 800 \mathrm{~mL}$. Non-responders requiring laparotomy were not experienced in the present case series.

In clinical backgrounds (Table 1), median shock index was 0.95 , median serum $\beta$-hCG value was 13,651 U/L, and median hemoglobin value was $6.5 \mathrm{~g} / \mathrm{dL}$ at triage. The surgical diagnosis (Table 2) included 7 ampullary (including 5 abortions and 2 ruptures), 5 ruptured isthmic (including 1 heterotopic pregnancy in remnant tube after salpingectomy), 4 ruptured interstitial, and 2 ruptured ovarian pregnancies. Among these cases initially managed by gasless LESS surgery, conversion to multi-port laparoscopic surgery was required due to difficult hemostasis (Table 2) in two cases with ruptured interstitial pregnancy. Furthermore, bank blood was transfused in addition to intraoperative autologous blood transfusion (Table 3) in these cases. Laparotomic conversion was not experienced in the present case series.

Surgical procedures in 16 cases successfully completed by gasless LESS surgery (Table 2) included unilateral salpingectomy for 7 ampullary and 5 isthmic pregnancies, cornuotomy and cornual resection for each 1 case with interstitial pregnancy, and unilateral salpingo-oophorectomy and partial ovarian resection for each 1 case with ovarian pregnancy.

In surgical outcomes of 16 cases successfully completed by gasless LESS surgery (Table 3), median surgical duration was $57.5 \mathrm{~min}$, median amounts of salvaged autologous blood were $950 \mathrm{~mL}$, median amounts of donated autologous blood were $540 \mathrm{~mL}$, and median hemoglobin value on postoperative day 1 was $9.6 \mathrm{~g} / \mathrm{dL}$. The median $\triangle \mathrm{Hb}$ values before and after LESS surgery (on postoperative day 1 ) were $+2.5 \mathrm{~g} / \mathrm{dL}$ (range $+0.4 \pm 6.1 \mathrm{~g} / \mathrm{dL}$ ).

The degree of hemoperitoneum was well correlated with the shock index $\left(R^{2}=0.59, P<0.05\right)$ (Fig. 2).

Surgical complications included three persistent ectopic pregnancy requiring systemic MTX administration (16.7\%) and 1 umbilical port-site infection (5.6\%). Additional systemic MTX administration was required 2 times in one ampullary pregnancy, 3 times in one interstitial pregnancy and once in one ovarian pregnancy. Adverse effects associated with the use of intraoperative cell salvage [7] were not observed.

\section{Discussion}

When a woman-especially one of childbearing agepresents with hypovolemic shock with an acute abdominal event, it represents a diagnostic and therapeutic challenge that requires immediate intervention with a 
Table 1 The clinical backgrounds of ectopic pregnancies with significant hemoperitoneum $\geq 800 \mathrm{~mL}(n=18)$

\begin{tabular}{lc}
\hline Variables & Values \\
\hline Age (years, median [range]) & $30.5(22-40)$ \\
Body mass index ( $\mathrm{kg} / \mathrm{m}^{2}$, median [range]) & $21(18.1-28.0)$ \\
Parity (number, median [range]) & $1(0-3)$ \\
Previous abdominal surgery (number, \%) & $1(5.6 \%)$ \\
$\quad$ Cesarean section & $1(5.6 \%)$ \\
Laparoscopic salpingectomy for hydrosalpinx & $1(5.6 \%)$ \\
Laparoscopic salpingectomy for tubal pregnancy & $15(83.3 \%)$ \\
$\quad$ None & $0.95(0.55-1.92)$ \\
Shock index at triage (heart rate/systolic blood pressure, median [range]) & $1(5.6 \%)$ \\
Class I: SI <0.6 (number, \%) & $10(55.6)$ \\
Class II: SI $\geq 0.6$ to $<1.0$ (number, \%) & $4(22.2)$ \\
Class III SI $\geq 1.0$ to < 1.4 (number, \%) & $3(16.7)$ \\
Class IV: SI $\geq 1.4$ (number, \%) & $7(5-10)$ \\
Estimated gestational weeks (median [range]) & 13,651 (1147-50,736) \\
Serum $\beta$-hCG (U/L, median [range]) & $6.5(2.2-8.9)$ \\
\hline Hemoglobin value at triage (g/dL, median [range]) &
\end{tabular}

$\beta$-hCG $=\beta$-human chorionic gonadotropin

multi-disciplinary approach, to avoid significant morbidity and potential mortality [2].

Once significant peritoneal hemorrhage is identified during intervention, and homologous blood transfusion is the most prompt and established way to achieve cardiovascular stability, which is necessary for the treatment of the causative disorder [3]. On the other hand, homologous blood transfusion may be associated with potential risks, including transfusion reactions caused by blood mismatch, and the transmission of blood-borne infections, including human immunodeficiency viruses, hepatitis viruses, and prions $[6,7]$.
Furthermore, when bank blood is not readily available due to limited resources or because the patient is positive for irregular antibodies, or when religious objections to the transfusion of homologous blood are expressed, the transfusion of homologous blood may not be appropriate, even in the emergency setting [6, 7].

As an acceptable alternative to resolve the negative effects caused by homologous blood transfusion, intraoperative autologous blood transfusion is considered to be a feasible option that can be applied in critical situations when immediate blood transfusion is required in the perioperative setting [2].

Table 2 The surgical diagnosis and procedures of 16 and 2 ectopic pregnancies with significant hemoperitoneum $\geq 800 \mathrm{~mL}$, managed by gasless laparoendoscopic single-site surgery and conversion to multi-port laparoscopic surgery, respectively

\begin{tabular}{lll}
\hline Variables & Gasless laparoendoscopic single-site surgery $(n=16)$ & Conversion to multi-port laparoscopic surgery $(n=2)$ \\
\hline Surgical diagnosis (number, \%) & & 0 \\
Ampullary pregnancy & $7(43.8 \%)$ & 0 \\
Isthmic pregnancy & $5(31.3 \%)^{\mathrm{a}, \mathrm{b}}$ & $2(100 \%)^{\mathrm{c}}$ \\
Interstitial pregnancy & $2(12.5 \%)$ & 0 \\
Ovarian pregnancy & $2(12.5 \%)$ & 0 \\
Surgical procedures (number, \%) & & 0 \\
Unilateral salpingectomy & $12(75.0 \%)$ & 0 \\
Unilateral salpingo-oophorectomy & $1(6.25 \%)$ & $1(50 \%)$ \\
Partial ovarian resection & $1(6.25 \%)$ & $1(50 \%)$ \\
Cornuotomy & $1(6.25 \%)$ & \\
Cornual resection & $1(6.25 \%)$ &
\end{tabular}

${ }^{\mathrm{a}}$ One case with heterotopic isthmic pregnancy in remnant tube after salpingectomy was included

${ }^{b}$ One case with isthmic pregnancy in remnant tube after salpingo-oophorectomy was included

${ }^{c}$ One case with interstitial pregnancy after salpingectomy was included 
Table 3 The surgical outcomes of ectopic pregnancies with significant hemoperitoneum $\geq 800 \mathrm{~mL}$, including 16 cases managed by gasless laparoendoscopic single-site surgery and 2 cases managed by conversion to multi-port laparoscopic surgery

\begin{tabular}{lll}
\hline Variables & $\begin{array}{l}\text { Gasless laparoendoscopic single-site surgery } \\
(n=16) \text { (median [range]) }\end{array}$ & $\begin{array}{l}\text { Conversion to multi-port laparoscopic } \\
\text { surgery }(n=2)(\text { value) }\end{array}$ \\
\hline Surgical duration (minutes) & $57.5(31-93)$ & 64,105 \\
Amounts of intraoperatively salvaged blood $(\mathrm{mL})$ & $950(800-2000)$ & 2340,1990 \\
Amounts of donated autologous blood $(\mathrm{mL})$ & $540(450-1300)$ & 980,680 \\
Amounts of transfused bank blood (units) & 0 & $4^{\mathrm{a}}, 6^{\mathrm{b}}$ \\
Hemoglobin value on postoperative day 1 (g/dL) & $9.6(7.7-10.9)$ & $8.2,10.7$ \\
$\begin{array}{l}\text { Postoperative hospital stay (days) } \\
\text { Surgical complications (number, \%) }\end{array}$ & $4(3-7)$ & 5,7 \\
$\begin{array}{l}\text { Persistent ectopic pregnancy requiring systemic methotrexate } \\
\text { administration }\end{array}$ & $3(18.8 \%)$ & 0 \\
Umbilical port-site infection & $1(6.3 \%)$ & 0 \\
\hline
\end{tabular}

${ }^{\mathrm{a}}$ Six units of fresh frozen plasma were additionally used

${ }^{\mathrm{b}}$ Ten units of fresh frozen plasma and 10 units of platelet concentrate were additionally used

In the present case series, although gasless LESS surgery was successfully completed without the use of homologous red blood cells and fresh frozen plasma, even in a case with $2000 \mathrm{~mL}$ of hemoperitoneum, the amount of hemoperitoneum that is appropriate for autologous salvage and transfusion needs to be practically determined from the viewpoint of costs and benefits because this procedure requires the installation of a specialized cell salvage device that must be operated by an experienced medical engineer, usually in an emergency situation [2].

Furthermore, since coagulation components, such as plasma, platelets, and coagulation factors are washed out by the Continuous Autotransfusion System [6, 7], coagulation disorders may influence the surgical outcomes by requiring conversion to multi-port laparoscopy or laparotomy [2], especially in cases needing complex surgical procedures such as suturing hemostasis for interstitial pregnancy [2], as occurred in the present case series.

In comparison to laparotomy, laparoscopic surgery with gasless abdominal wall-lifting [2] or pneumoperitoneum $[1,4,5]$ is considered to be a less invasive option for the management of ectopic pregnancy. Furthermore, with recent advances in techniques, LESS surgery has been suggested as a feasible and preferable alternative to conventional multi-port laparoscopy $[8,10-12]$, because of its potential advantages, which include easy anatomical access to the pelvic cavity through a single umbilical skin incision, a safer approach to the abdominal cavity by an open procedure, especially in patients with a

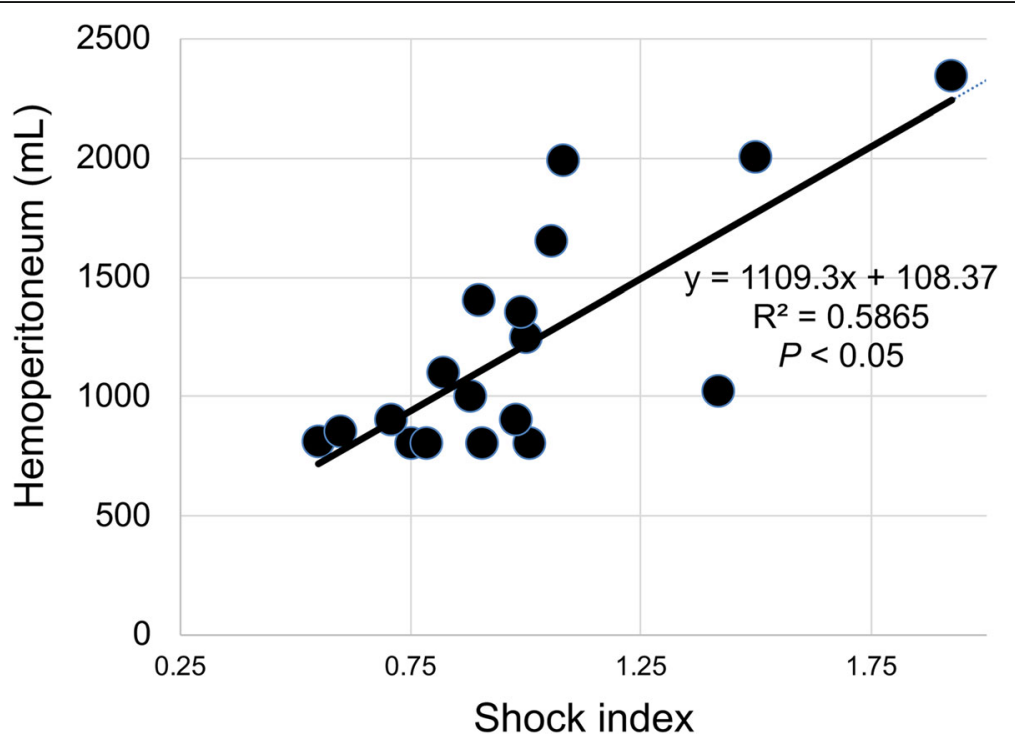

Fig. 2 Scatter plots of the degree of hemoperitoneum $(\mathrm{mL})$ versus the triage shock index, showing the positive Pearson correlation 
history of previous abdominal surgery, and cosmetic results obtained as the procedure involves a single surgical wound limited to the umbilicus $[8,10-12]$.

Although a wider incision in single-port laparoscopy may allow better visualization in comparison to a 5- to 12-mm incision in multi-port laparoscopy [11], there may exist some technical difficulties in manipulation through a single incision, especially when complex procedures, such as suturing hemostasis, are required $[8$, 12]. Actually, because all of the surgical instruments are introduced through a single incision, they may impede each other and occasionally collide; thus, even gynecologic surgeons with sufficient laparoscopic experience must adapt to inline placement and the manipulation of the surgical instruments.

Reports on the use of LESS surgery for the management of ectopic pregnancies with significant hemoperitoneum are limited $[8,9,11]$; however, the present results were obtained from such a case series after appropriate selection. Thus, the major strength of the present study is its thorough description of the combined application of relatively new therapeutic techniques-gasless LESS surgery [8] and intraoperative autologous blood cell salvage and donation $[2,6,7]$ - which were performed by the same gynecologic surgeons with sufficient experience in minimally invasive procedures.

On the other hand, the present study only represents the retrospective outcomes from a limited number of cases due to rarity and should not be constructed as a report on the superiority of gasless LESS surgery over either LESS surgery with pneumoperitoneum or conventional multi-port laparoscopic surgery. Rather, we consider that gasless LESS surgery is a variable as a minimally invasive surgical option that can be one approach to lessen the technical difficulties in performing LESS surgery for management of ectopic pregnancy with significant hemoperitoneum.

Thus, given our management with success rate of $89 \%$ (16/18 cases) by gasless LESS surgery without the use of homologous blood transfusion, the present evaluation of gasless LESS surgery for the management of ectopic pregnancies with intraoperative autologous blood transfusion is important and of great clinical relevance.

However, since persistent ectopic pregnancy was observed in 3 cases requiring MTX administration, and in one case with umbilical port infection, further accumulation of cases is required to assess the potential value of LESS surgery with intraoperative autologous blood transfusion in ectopic pregnancies with significant hemoperitoneum.

In conclusion, this small case series emphasizes that, in appropriately selected cases, gasless LESS surgery with intraoperative autologous blood transfusion can be a safe and feasible alternative to multi-port laparoscopic surgery for the management of ectopic pregnancy even with significant hemoperitoneum. With the further accumulation of cases and discovery of potential beneficial effects, gasless LESS surgery is expected to be recognized not only as a safe and feasible option but also as a better approach for the treatment of ectopic pregnancy with significant hemoperitoneum.

\section{Abbreviations \\ CRP: C-reactive protein; LESS: Laparoendoscopic single-site; MTX: Methotrexate; $\beta$-hCG: $\beta$-Human chorionic gonadotropin}

\section{Funding}

No supplemental funding was provided for this research.

\section{Availability of data and materials}

The datasets used and/or analyzed during the current study are available from the corresponding author on reasonable request.

\section{Authors' contributions}

AT contributed to the project development, data collection, and manuscript writing. ST, MS, SS, and HN contributed to the data collection. All authors read and approved the final manuscript.

\section{Ethics approval and consent to participate}

This study was approved by the Institutional Review Board of Gifu Prefectural Tajimi Hospital (IRB Number: 2018-04-1). All procedures performed in studies involving human participants were in accordance with the ethical standards of the institutional and/or national research committee and with the 1964 Helsinki declaration and its later amendments or comparable ethical standards. Each patient and her family signed a surgical informed consent form for the relevant procedures after an explanation that laparotomy and use of blood products including homologous red blood cells and fresh frozen plasma might be required if the proposed therapy failed to manage the gynecologic emergencies. If the patient was assumed to have difficulty in understanding her condition due to symptoms such as severe pain or hemodynamic instability, explanation was given only to her family.

\section{Consent for publication}

Written permission for publication of their cases was obtained. Women who did not consent to have their clinical information used for research purposes were excluded from the study cohorts.

\section{Competing interests}

The authors declare that they have no competing interests.

\section{Publisher's Note}

Springer Nature remains neutral with regard to jurisdictional claims in published maps and institutional affiliations.

Received: 19 October 2018 Accepted: 19 February 2019

Published online: 05 March 2019

\section{References}

1. Odejinmi F, Rizzuto I, Oliver R, Alalade A, Agarwal N, Olowu O (2015) Beyond guidelines: effectiveness of a programme in achieving operative laparoscopy for all women requiring surgical management of ectopic pregnancy. Gynecol Obstet Investig 80:46-53 https://doi.org/10.1159/ 000371763.

2. Takeda A, Manabe S, Mitsui T, Nakamura H (2006) Management of patients with ectopic pregnancy with massive hemoperitoneum by laparoscopic surgery with intraoperative autologous blood transfusion. J Minim Invasive Gynecol 13:43-48 https://doi.org/10.1016/j.jmig.2005.09.100.

3. Huang J, Qin D, Gu C, Huang Y, Ma H, Huang H, Huang F, Ruan J, Ling M (2017) Autologous and nonautologous blood transfusion in patients with ruptured ectopic pregnancy and severe blood loss. Biomed Res Int 2017: 7501807 https://doi.org/10.1155/2017/7501807.

4. Cohen A, Almog B, Satel A, Lessing JB, Tsafrir Z, Levin I (2013) Laparoscopy versus laparotomy in the management of ectopic pregnancy with massive 
hemoperitoneum. Int J Gynaecol Obstet 123:139-141 https://doi.org/10. 1016/.i.jgo.2013.05.014.

5. Beuran M, Negoi I, Hostiuc S, Catena F, Sartelli M, Negoi Rl, Paun S (2016) Laparoscopic approach has benefits in gynecological emergencies - even for massive hemoperitoneum. Chirurgia (Bucur) 111:48-53.

6. Carless PA, Henry DA, Moxey AJ, O'Connell D, Brown T, Fergusson DA (2010) Cell salvage for minimising perioperative allogeneic blood transfusion. Cochrane Database Syst Rev CD001888. https://doi.org/10.1002/14651858. CD001888.pub3.

7. DeAndrade D, Waters JH, Triulzi DJ, Alarcon L, Wisniewski MK, Dyga R, Yazer MH (2016) Very low rate of patient-related adverse events associated with the use of intraoperative cell salvage. Transfusion 56:2768-2772 https://doi. org/10.1111/trf.13791.

8. Takeda A, Imoto S, Mori M, Nakano T, Nakamura H (2011) Early experience with isobaric laparoendoscopic single-site surgery using a wound retractor for the management of ectopic pregnancy. Eur J Obstet Gynecol Reprod Biol 154:209-214 https://doi.org/10.1016/..ejogrb.2010.10.006.

9. Takeda A, Hayashi S, Imoto S, Sugiyama C, Nakamura H (2014) Pregnancy outcomes after emergent laparoscopic surgery for acute adnexal disorders at less than 10 weeks of gestation. J Obstet Gynaecol Res 40:1281-1287 https://doi.org/10.1111/jog.12332.

10. Karasu Y, Akselim B (2018) Feasibility of single-incision laparoscopy for ruptured ectopic pregnancies with hemoperitoneum. Minim Invasive Ther Allied Technol 9:1-5 https://doi.org/10.1080/13645706.2018.1470538.

11. Yang J, Na YJ, Song YJ, Choi OH, Lee SK, Kim HG (2016) The effectiveness of laparoendoscopic single-site surgery (LESS) compared with conventional laparoscopic surgery for ectopic pregnancy with hemoperitoneum. Taiwan J Obstet Gynecol 55:35-39 https://doi.org/10.1016/j.tjog.2015.02.005.

12. Takeda A, Imoto S, Mori M, Yamada J, Nakamura H (2013) Management of large adnexal tumors by isobaric laparoendoscopic single-site surgery with a wound retractor. Eur J Obstet Gynecol Reprod Biol 66:185-189 https://doi. org/10.1016/j.ejogrb.2012.10.016.

\section{Submit your manuscript to a SpringerOpen ${ }^{\circ}$ journal and benefit from:}

- Convenient online submission

- Rigorous peer review

- Open access: articles freely available online

- High visibility within the field

- Retaining the copyright to your article

Submit your next manuscript at $\boldsymbol{\nabla}$ springeropen.com 